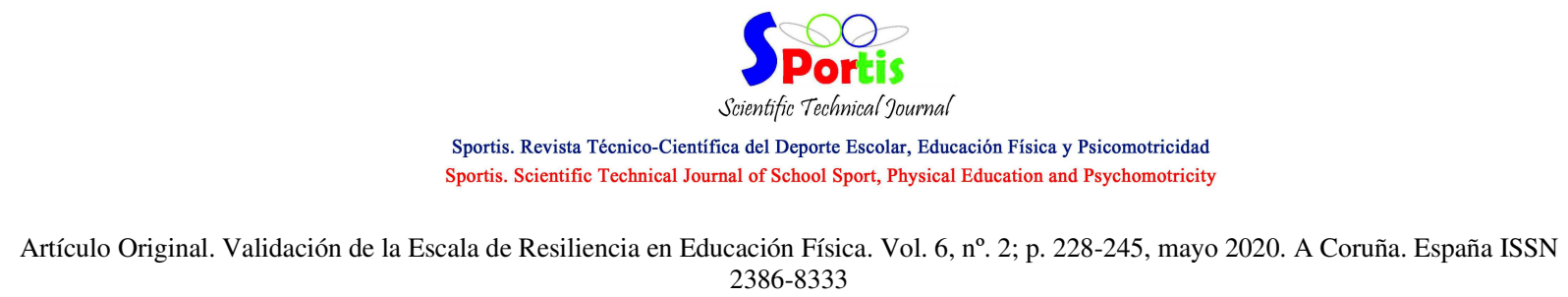

\title{
Validación de la Escala de Resiliencia en Educación Física
}

\section{Validation of the resilience scale to Physical Education classes}

Rubén Trigueros ${ }^{1}$, Noelia Navarro ${ }^{1}$, José Manuel Aguilar-Parra ${ }^{1, *}$, Rosario Bermejo ${ }^{2}$; Carmen Ferrandiz ${ }^{2}$.

${ }^{1}$ Departamento de Psicología, Facultad de Ciencias de la Educación, Universidad de

\author{
Almería \\ ${ }^{2}$ Departamento de Psicología Evolutiva y de la Educación, Facultad de Ciencias de la \\ Educación, Universidad de Murcia
}

* Correspondencia a: Dr. José M. Aguilar-Parra. Departamento de Psicología, Edificio Departamental de Humanidades y Ciencias de la Educación I (Edif. A), Universidad de Almería, Ctra. Del Sacramento s/n La Cañada, Almería 04120, España. Tel: +34 9500145376. E-mail: jmaguilar@ual.es

Cronograma editorial: Artículo recibido: 11/04/2019 Aceptado: 07/04/2020 Publicado: 01/05/2020 DOI: https://doi.org/10.17979/sportis.2020.6.2.5245 


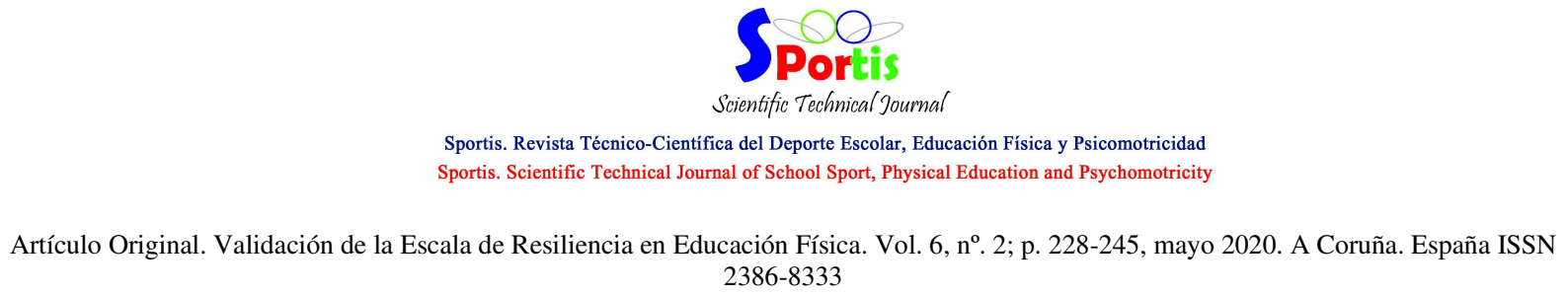

\title{
Resumen
}

Las clases de Educación Física se caracterizan por la continua exposición por parte del alumnado de una serie de circunstancias adversas y estresantes ante las cuales en mayor o menor medida deben de enfrentarse en algún momento. Por ello, el objetivo de este estudio fue validar y adaptar la Escala de Resiliencia en el contexto Deportivo de Trigueros, Álvarez, Aguilar-Parra y Rosado (2017) al contexto de la Educación Física. En el estudio participaron 515 estudiantes de secundaria con edades comprendidas entre los 13 y 19 años $(M=15.65$; $D T=1.28)$ pertenecientes a varios centros educativos del sur-este de España. El análisis factorial exploratorio y confirmatorio reveló unos índices de ajuste adecuados para la nueva versión de la escala, mostrándose la estructura factorial invariante respecto al generó. Los dos factores que integran la escala, competencia personal y aceptación de uno mismo y de la vida, obtuvieron una alta consistencia interna y estabilidad temporal. La versión española de la Escala de Resiliencia en el contexto Educación Física se mostró como un instrumento con adecuadas propiedades psicométricas para medir la resiliencia en el contexto de la Educación Física en adolescentes.

\section{Palabras clave}

Resiliencia; Educación Física; adolescencia; análisis factorial.

\begin{abstract}
Physical Education classes are characterized by the continuous exposure of students to a series of adverse and stressful circumstances which they must face at some point. Therefore, the objective of this study was to validate and adapt the Resilience Scale in the Sport context of Trigueros, Álvarez, Aguilar-Parra and Rosado (2017) to the context of Physical Education. The study involved 515 high school students aged between 13 and $19(\mathrm{M}=15.65, \mathrm{SD}=1.28)$ belonging to several educational centers in the south-east of Spain. The exploratory and confirmatory factor analysis revealed adequate adjustment indices for the new version of the scale, showing the factorial structure invariant with respect to the generated one. The two factors that make up the scale, personal competence and acceptance of oneself and life, obtained a high internal consistency and temporary stability. The Spanish version of the Resilience Scale in the Physical Education context was shown as an instrument with adequate psychometric properties to measure resilience in the context of Physical Education in adolescents.
\end{abstract}

\section{Keywords}

Resilience; physical education; teenagers; factorial analysis.

\section{Introducción}

Para citar este artículo utilice la siguiente referencia: Trigueros, R.; Navarro, N.; Aguilar-Parra, J.M.; Bermejo, R.; Ferrandiz, C. (2020). Validación de la Escala de Resiliencia en Educación Física. Sportis Sci J, 6 (2), 228-245. DOI: https://doi.org/10.17979/sportis.2020.6.2.5245 


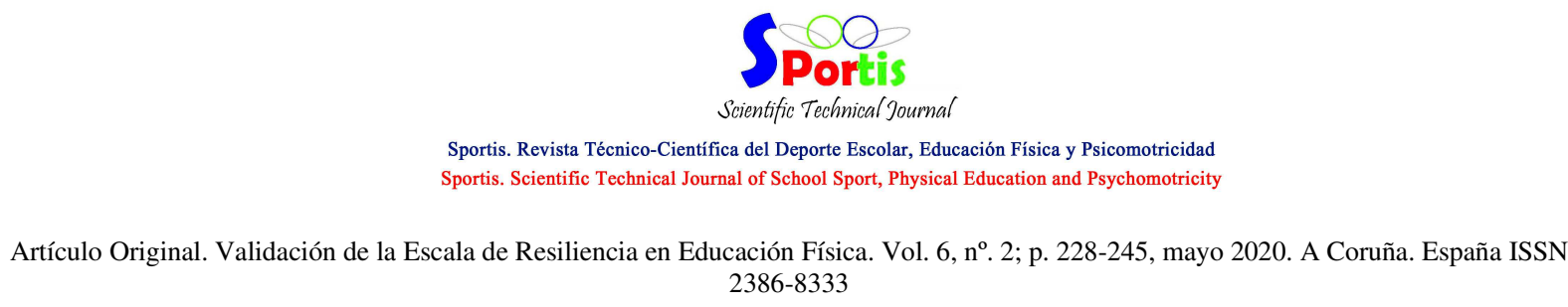

Entre las principales características de las clases de Educación Física se encuentra la continua exposición por parte del alumnado de una serie de circunstancias adversas y potencialmente estresantes ante las cuales en mayor o menor medida deben de enfrentarse en algún momento (Kumar y Bhukar, 2013; Sundblad, Jansson, Saartok, Renström y Engström, 2008). De esta forma, los estudiantes a lo largo de su periodo académico deben de enfrentarse a una serie tareas que les suponga una gran exigencia motriz, fracaso en los exámenes, roces con los compañeros, situaciones embarazosas o lesiones (Bernstein, Phillips y Silverman, 2011; Lonsdale, Rosenkranz, Peralta, Bennie, Fahey y Lubans, 2013). Es por ello, que a partir de la vivencia de este tipo de situaciones se da una respuesta ante la situación adversa que reconduce a la persona hacia un nivel normal de funcionamiento (Southwick, Bonanno, Masten, Panter-Brick

y Yehuda, 2014).

Son precisamente estas situaciones adversas y su consiguiente superación lo que desde la metateoría de la resiliencia (Richardson, 2002; Tavakolizadeh, Yazdi y Akbary, 2019) se ha tratado de explicar. Esta teoría afirma que la resiliencia comienza en una situación de equilibrio físico, mental y espiritual que se ve interrumpido cuando se presenta una determinada situación donde el individuo no posee los recursos o habilidades suficientes para enfrentarse ante situaciones adversas (Richardson, 2002). Pasado el tiempo, el individuo se reajustará y volverá a alcanzar el equilibrio elevando su nivel de resiliencia u homeostasis. Sin embargo, esta metateoría presenta una serie de limitaciones, ya que considera la resiliencia como un modelo lineal, donde un solo evento se contrapone al individuo (Richardson, 2002). Además, el modelo no explica cómo las emociones pueden afectar al proceso de superación. Esto resulta de especial importancia dado el carácter protector que tienen las emociones en las conductas de los individuos (Bamber y Schneider, 2016) y que aquellas personas que muestran una capacidad de resiliencia valoran las emociones como facilitadoras para alcanzarla (Fletcher y Sarkar, 2013). Dadas estas limitaciones, Fletcher y Sarkar (2012) reconceptualizaron la noción de resiliencia como aquella asociada con la posesión y presencia de factores de vulnerabilidad y factores de protección dentro y fuera del individuo que influyen en la adaptación positiva hacia el riesgo.

Para citar este artículo utilice la siguiente referencia: Trigueros, R.; Navarro, N.; Aguilar-Parra, J.M.; Bermejo, R.; Ferrandiz, C. (2020). Validación de la Escala de Resiliencia en Educación Física. Sportis Sci J, 6 (2), 228-245. DOI: https://doi.org/10.17979/sportis.2020.6.2.5245 


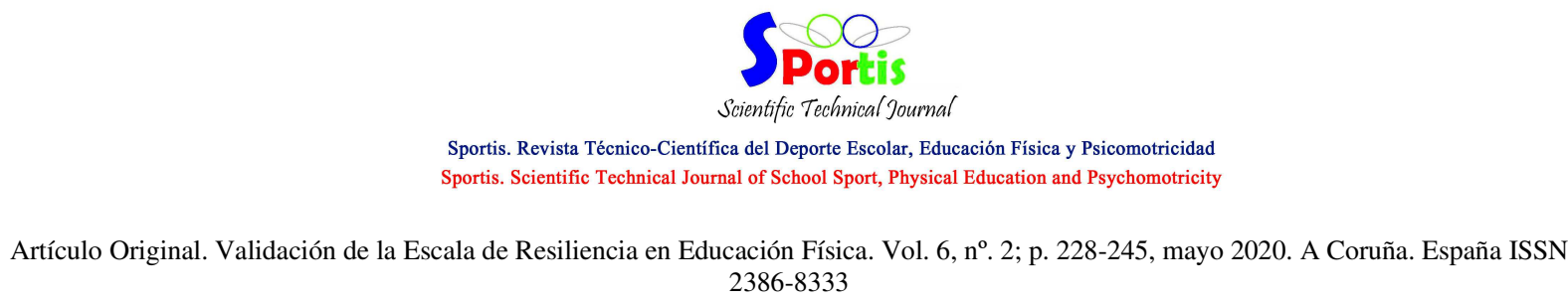

Las investigaciones sobre la resiliencia se han centrado principalmente en el campo de la salud (Heath, Donald, Theron y Lyon, 2014), el militar (Thompson y Dobbins, 2018), el laboral (Vanhove, Herian, Perez, Harms y Lester, 2016), estilo de vida (Robertson y Cooper, 2013) y el deportivo (Bryan, O'Shea y MacIntyre, 2019), existiendo apenas estudios que se hayan centrado en el ámbito educativo. En este sentido, los estudios relacionados con la resiliencia han mostrado una relación positiva hacia el afrontamiento de acontecimientos estresantes (Oliva, Jiménez, Parra y Sánchez, 2008), la calidad de vida (Robertson y Cooper, 2013), la regulación emocional (Min, Yu, Lee y Chae, 2013), inteligencia emocional (Schneider, Lyons y Khazon, 2013) y motivación (Resnick, 2018). Por otro lado, la resiliencia se ha asociado de forma negativa con los estados emocionales negativos (e.g., ansiedad, depresión; Robertson y Cooper, 2013) y hacia las conductas de riesgo (McNair, Gipson, Denson, Thompson, Sutton y Hickson).

Los instrumentos que se han utilizado para evaluar la resiliencia en la población general han sido múltiples, como la Connor-Davidson Resilience Scale (Connor y Davidson, 2003), The Baruth Protective Factors Inventory (Baruth y Carroll, 2002) o The Adolescent Resilience Scale (ARS; Oshio, Kaneko, Nagamine y Nakaya, 2003), aunque dichas herramientas se elaboraron a partir de una metodología de rasgo (Richardson, 2002). Otra herramienta para evaluar la resiliencia y que ha sido desarrollada y ampliamente utilizada ha sido The Resilience Scale validada por Wagnild y Young (1993), esta herramienta consta de dos factores que permiten conocer las características de la personalidad positiva: la competencia personal, definida como el reconocimiento de la capacidad personal, independencia, perseverancia, habilidad, y la aceptación de uno mismo y de la vida, como equivalente a adaptación y flexibilidad. Los estudios que la han utilizado (Lundman, Strandberg, Eisemann, Gustafson y Brulin, 2007; Madewell y Ponce-García, 2016; Rodríguez, Pereyra, Gil, Jofré, De Bortoli y Labiano, 2009), tanto con población clínica, como no clínica y en diferentes rangos de edades, encontrándose en todos ellos adecuadas propiedades de fiabilidad y consistencia interna (Wagnild, 2009).

Esta herramienta ha sido adaptada al ámbito deportivo (Cardoso y Sacomori, 2014; Trigueros, Álvarez, Aguilar-Parra y Rosado, 2017), no existiendo ningún estudio que haya proporcionado una medida de la resiliencia para su uso en la EF. En este sentido, el contexto

Para citar este artículo utilice la siguiente referencia: Trigueros, R.; Navarro, N.; Aguilar-Parra, J.M.; Bermejo, R.; Ferrandiz, C. (2020). Validación de la Escala de Resiliencia en Educación Física. Sportis Sci J, 6 (2), 228-245. DOI: https://doi.org/10.17979/sportis.2020.6.2.5245 


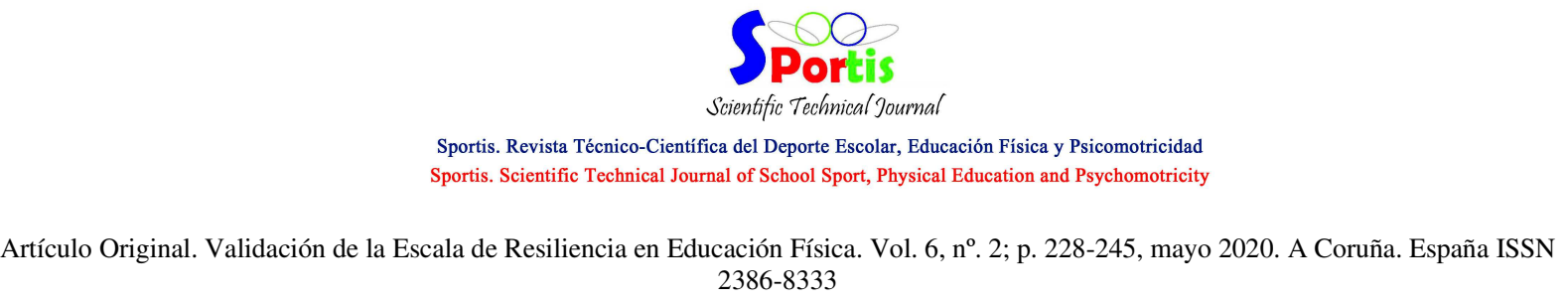

deportivo como el contexto de la EF tiene ciertos elementos comunes relacionados con la exigencia física, alcanzar el éxito o el fracaso y las relaciones con los compañeros (Kohl y Cook, 2013). En consecuencia, el propósito del presente estudio es adaptar y validar The Resilience Scale de Wagnild y Young (1993) tomando como referencia la Escala de Resiliencia en el Contexto Deportivo de Trigueros et al, (2017a). De esta forma, el objetivo del presente estudio fue validar y adaptar la Escala de Resiliencia en el contexto Deportivo de Trigueros et al, (2017) al contexto de la Educación Física con el fin de obtener una herramienta válida para los contenidos propios de la EF en la etapa de Educación Secundaria y Bachiller, ya que resiliencia es considerada como un atributo que contribuye en la capacidad de adaptación del individuo ante las posibles vicisitudes que puedan enfrentarse, generando con ello comportamientos adaptativos positivos propios del área de EF.

\section{Método}

\section{Participantes}

En el presente estudio tomaron parte 515 estudiantes de Educación Secundaria (274 hombres y 241 mujeres), con edades comprendidas entre los 13 y 19 años $(M=15.65 ; D T=$ 1.28), pertenecientes a la provincia de Almería. Para el análisis de estabilidad temporal, tomaron parte 58 sujetos (32 hombres y 26 mujeres), con edades comprendidas entre los 14 y 16 años $(M=15.3 ; D T=.61)$.

\section{Procedimiento}

Para poder llevar a cabo la validación de la Escala de Resiliencia en el contexto de EF, se realizó una adaptación de la Escala de Resiliencia en el Contexto Deportivo de Trigueros et al, (2017a) contextualizando los factores que hacen mención a los elementos más específicos del deporte y los poco frecuentes en EF, como puede ser el factor de aceptación de sí mismo y de la vida que fue modificado por aceptación de sí mismo y del contexto. Esta decisión fue tomada por un grupo de expertos en la materia formada por tres profesores de EF y dos expertos psicólogos con más de 10 años de experiencia en el estudio de la resiliencia. Además, se realizaron modificaciones en la redacción de varios ítems, sustituyendo alusiones al aspecto competitivo del ámbito deportivo y conceptos personales difíciles de entender, dada la falta de madurez de los adolescentes, por las tareas propias del área de EF.

\footnotetext{
Para citar este artículo utilice la siguiente referencia: Trigueros, R.; Navarro, N.; Aguilar-Parra, J.M.; Bermejo, R.; Ferrandiz, C. (2020). Validación de la Escala de Resiliencia en Educación Física. Sportis Sci J, 6 (2), 228-245. DOI: https://doi.org/10.17979/sportis.2020.6.2.5245 


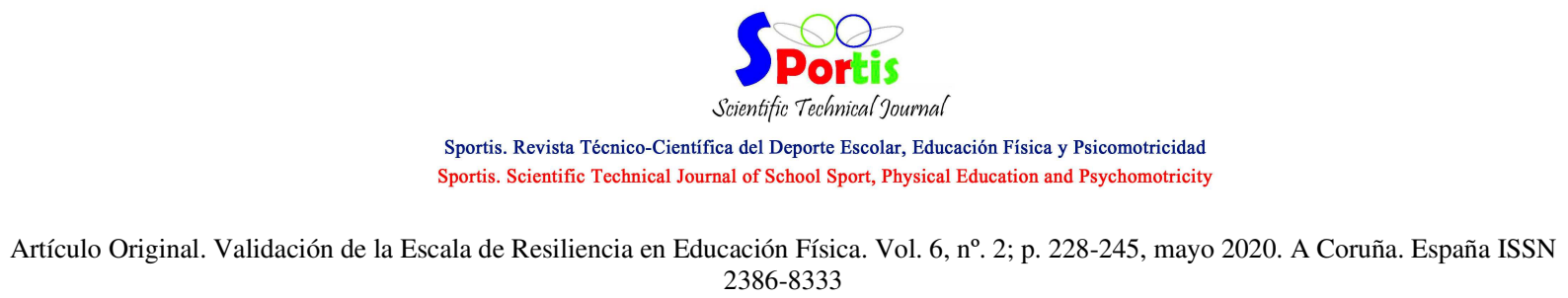

Una vez obtenido el cuestionario se contactó con diversos centros educativos de la provincia de Almería, a los que también se les informó del objetivo de la investigación y solicitó su colaboración. A los sujetos se le requirió una autorización paterna para su participación dada su minoría de edad. Antes de administrar la escala a la totalidad de los participantes, ésta fue cumplimentada por un pequeño grupo de personas para asegurar la correcta comprensión de todos los ítems. La administración de la escala se llevó a cabo insistiendo en el anonimato y en que no existían contestaciones verdaderas o falsas, sino que simplemente se les preguntaba por sus propios pensamientos. El tiempo estimado para completar el cuestionario estuvo en torno a 10 minutos.

El estudio se llevó acabo en cumplimiento de las directrices de la Asociación Psicológica Americana. El Comité de Bioética de Investigación de la Universidad de Almería, España, aprobó el presente estudio (Ref. UAL BIO 2019/014).

\section{Instrumentos de medida}

Resiliencia. Se utilizó la versión elaborada según se detalla en el apartado del procedimiento. Este cuestionario consta de 25 ítems repartidos entre dos factores: competencia personal, 17 ítems; y aceptación de sí mismo y del contexto, 8 ítems. Los sujetos debían indicar su respuesta por medio de una escala Likert de 1 (totalmente en desacuerdo) a 7 (totalmente de acuerdo).

Motivación Intrínseca. Se utilizó el factor que tiene el mismo nombre proveniente de la versión validada y adaptada de la Perceived Locus of Causality Revised (PLOC-R) de Vlachopoulos, Katartzi, Kontou, Moustaka y Goudas (2011) al contexto español de EF por Trigueros, Sicilia, Alcaraz-Ibáñez y Dumitru (2017b). La escala estuvo precedida por el encabezamiento "Participo en clase de Educación Física..." y consta de 4 ítems. Los estudiantes respondieron utilizando una escala Likert que oscilaba de 1 (nada verdadero) a 7 (totalmente verdadero).

\section{Análisis de datos}

Con el fin de determinar la validez y fiabilidad de la escala de Resiliencia en el contexto de la EF se analizaron sus propiedades psicométricas. En primer lugar, se realizó análisis estadísticos descriptivos y se testó la fiabilidad del instrumento a través de análisis de consistencia interna (alfa de Cronbach) y un análisis de estabilidad temporal (índice de

Para citar este artículo utilice la siguiente referencia: Trigueros, R.; Navarro, N.; Aguilar-Parra, J.M.; Bermejo, R.; Ferrandiz, C. (2020). Validación de la Escala de Resiliencia en Educación Física. Sportis Sci J, 6 (2), 228-245. DOI: https://doi.org/10.17979/sportis.2020.6.2.5245 


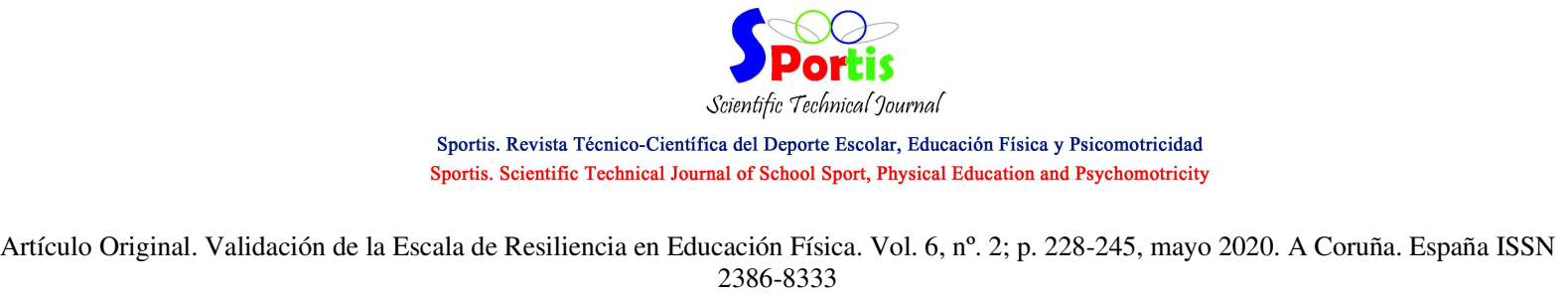

correlación intra-clase, CCI). En segundo lugar, se realizó un análisis factorial exploratorio (AFE) y un análisis factorial confirmatorio (AFC) con el fin de testar su estructura factorial. A continuación, se llevó a cabo un análisis multigrupo para analizar la invarianza respecto al género. En último lugar, se llevó a cabo un análisis de validez criterio a través de una prueba t. Para los análisis de datos se utilizaron los paquetes estadísticos SPSS 24.0 y AMOS 19.0.

Debido a que el coeficiente de Mardia resulto ser alto (83.89) para el AFC se utilizó el método de estimación de máxima verosimilitud junto con el procedimiento de bootstrapping. Los estimadores no resultaron afectados por la falta de normalidad, por lo que se considerados robustos (Byrne, 2001). Con el objetivo de aceptar o rechazar el modelo testado, se consideraron diversos índices de ajuste: $\chi 2$ /g1, CFI (Comparative Fit Index), IFI (Incremental Fit Index), RMSEA (Root Mean Square Error of Approximation) más su intervalo de confianza (IC) al 90\%, y SRMR (Standardized Root Mean Square Residual). Valores de $\chi^{2 / g l}$ inferiores a 3, valores para los índices incrementales (CFI e IFI) próximos o superiores a .95 y valores de RMSEA y SRMR inferiores o muy próximos a .06 y .08 se consideraron, respectivamente, como indicativos de un adecuado ajuste del modelo a los datos (Hair, Black, Babin, Anderson y Tatham, 2006).

\section{Resultados}

Análisis validez discriminante, correlaciones bivariadas y descriptivos.

La proporción HTMT de las correlaciones entre factores latentes (Tabla 1) fue de .41, sugiriendo la existencia de validez discriminante. Además, la tabla 2 muestra la correlación existente entre ambos factores siendo positiva entre ellos, lo que pone de manifiesto la clara reciprocidad entre ambos factores. Además, la puntuación media fue superior para aceptación de sí mismo y del contexto que para competencia personal. 


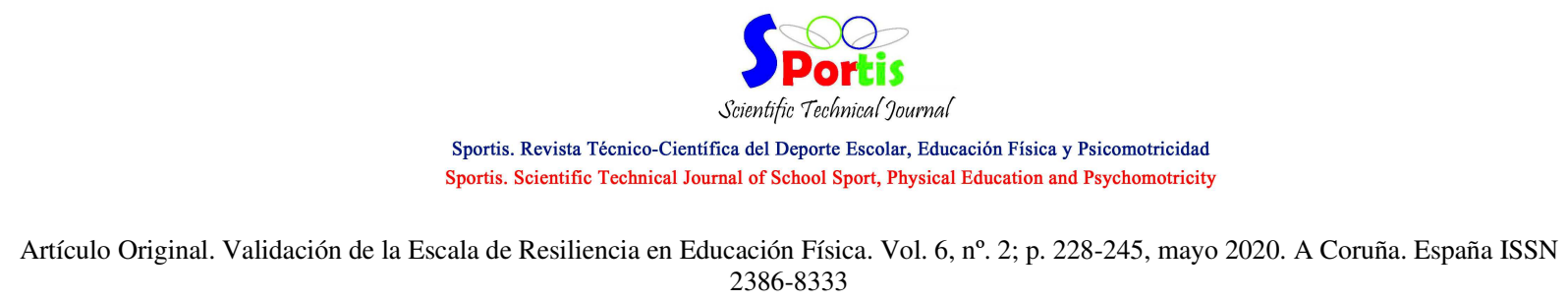

Tabla 1

Media, Desviación Típica, Correlaciones Bivariadas y Proporción HTMT de los Factores.

\begin{tabular}{|c|c|c|c|c|c|c|}
\hline Factores & $M$ & $D T$ & Rango & 1 & 2 & 3 \\
\hline 1. Competencia Personal & 4.62 & 1.26 & $1-7$ & & $.51 * *$ & $.53 * *$ \\
\hline $\begin{array}{l}\text { 2. Aceptación de sí mismo y del } \\
\text { contexto }\end{array}$ & 6.08 & 1.06 & $1-7$ & .41 & & $.41 * *$ \\
\hline 3. Motivación Intrínseca & 4.44 & 1.88 & $1-7$ & - & - & \\
\hline
\end{tabular}

Nota: Los valores bajo la diagonal corresponden a la proporción heterorrasgo-monorrasgo (HTMT) entre factores; $* * p<.001$.

\section{Análisis factorial exploratorio}

La tabla 1 muestra las correlaciones entre cada ítem y la puntuación total de la escala que se encontraba en un rango general entre .68 y .84. Estos resultados llevan a mantener todos los ítems, puesto que la correlación ítem-test es superior al punto de corte establecido en 0.30 (Fayers y Machin, 2000). El Alpha de Cronbach global fue de .82. Además, el análisis factorial exploratorio apoya la existencia de dos factores, mostrando un factor de saturación que oscilo entre .60 y .81 para competencia personal y entre .66 y .72 para aceptación de sí mismo y del contexto.

Tabla 2

Correlaciones entre cada ítem y la puntuación total de la escala.

\begin{tabular}{cccc}
\hline Ítems & Correlación ítem-test & $\begin{array}{c}\text { Alpha de Cronbach si } \\
\text { se elimina el ítem }\end{array}$ & $\begin{array}{c}\text { Factor de saturación de } \\
\text { cada ítem con su factor }\end{array}$ \\
\hline 1 & $.75^{* *}$ & .82 & $.63 \mathrm{~F} 1$ \\
2 & $.72 * *$ & .82 & $.74 \mathrm{~F} 1$ \\
3 & $.74 * *$ & .82 & $.78 \mathrm{~F} 1$ \\
4 & $.76^{* *}$ & .82 & $.79 \mathrm{~F} 1$ \\
5 & $.82 * *$ & .82 & $.81 \mathrm{~F} 1$ \\
\hline
\end{tabular}

Para citar este artículo utilice la siguiente referencia: Trigueros, R.; Navarro, N.; Aguilar-Parra, J.M.; Bermejo, R.; Ferrandiz, C. (2020). Validación de la Escala de Resiliencia en Educación Física. Sportis Sci J, 6 (2), 228-245. DOI: https://doi.org/10.17979/sportis.2020.6.2.5245 


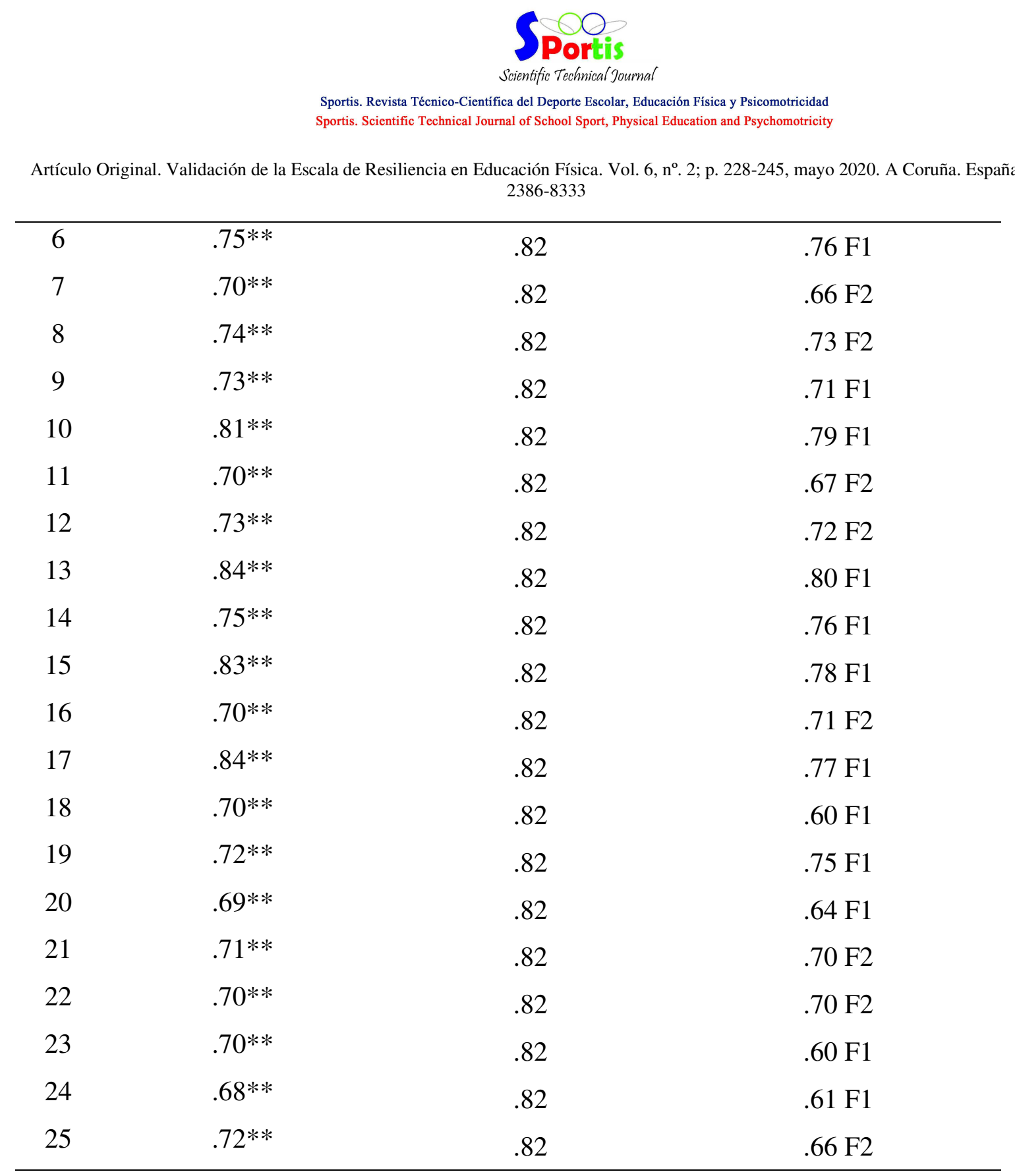

Nota: $\mathrm{F} 1=$ Competencia Personal; F2= Aceptación de sí mismo y del contexto; $* * p<.001$.

Análisis factorial confirmatorio

Los índices de ajuste del modelo testado (Figura 1) reveló unos índices de ajuste apropiados: $\chi^{2}(274 . N=515)=879.51, p<.001 ; \chi^{2} / g l=3.21 ; \mathrm{CFI}=.95 ;$ IFI $=.95$; RMSEA $=.06($ IC 90\% $=.060-.070) ;$ SRMR $=.049$. Los pesos de regresión estandarizados oscilaron entre .60 y .92 siendo estadísticamente significativos $(p<.001)$. En cuanto a la correlación entre los factores fue de .52 siendo estadísticamente significativo $(p<.001)$. 


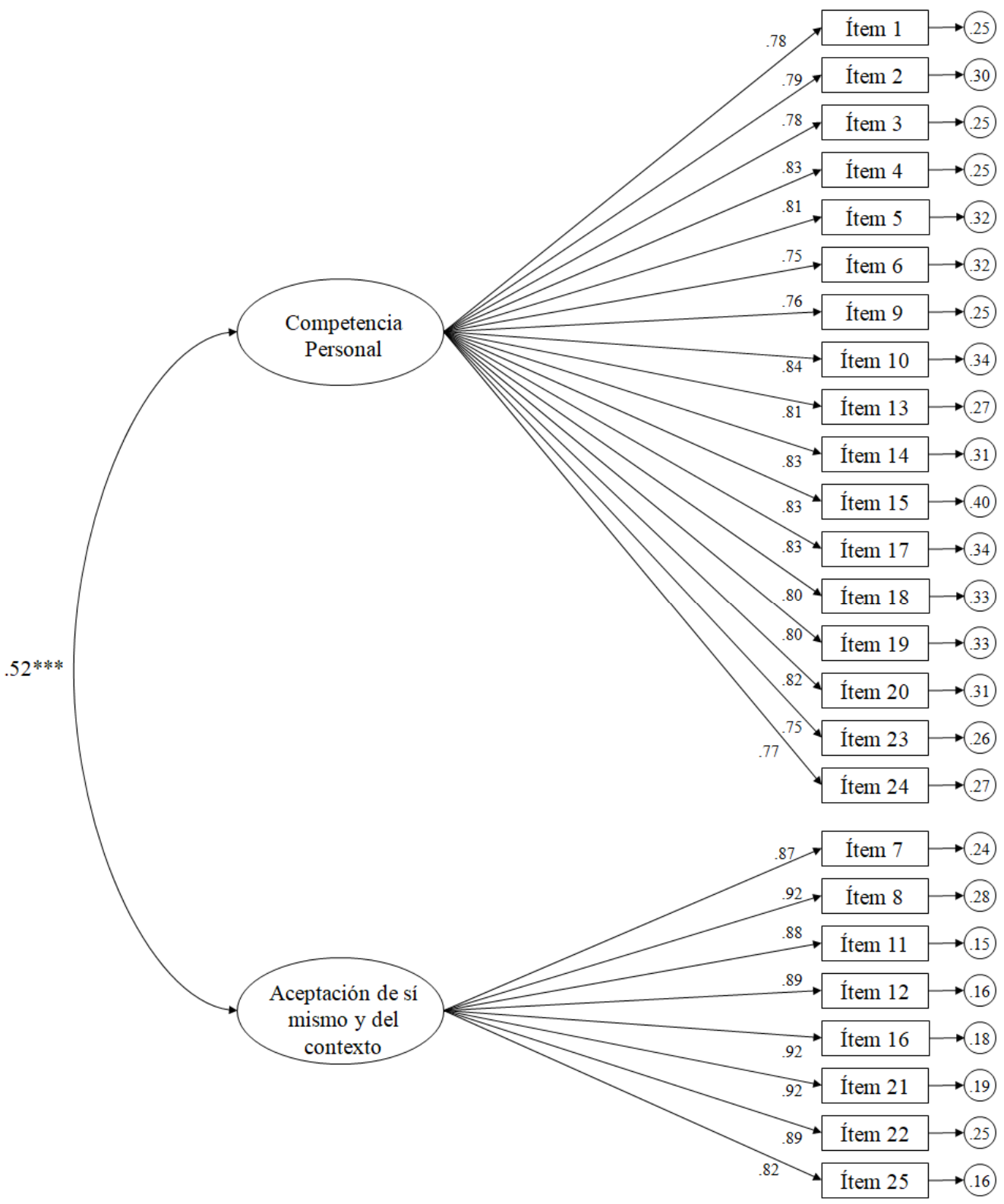

Figura 1. Análisis factorial confirmatorio de la EREF (Escala de Resiliencia en el contexto de la Educación Física). Las elipses representan los factores y los rectángulos representan los diferentes ítems. Las varianzas residuales se muestran en los círculos pequeños.

\section{Análisis de invarianza por género}

Con el fin de comprobar si la estructura factorial del modelo se muestra invariante respecto al género, se procedió a realizar un análisis multigrupo. Tal y como se muestra en la tabla 3 no se encontraron diferencias significativas entre el modelo 1 (modelo sin restricciones) y el modelo 2 (modelo de invarianza en los pesos de medida). Por su parte, los 


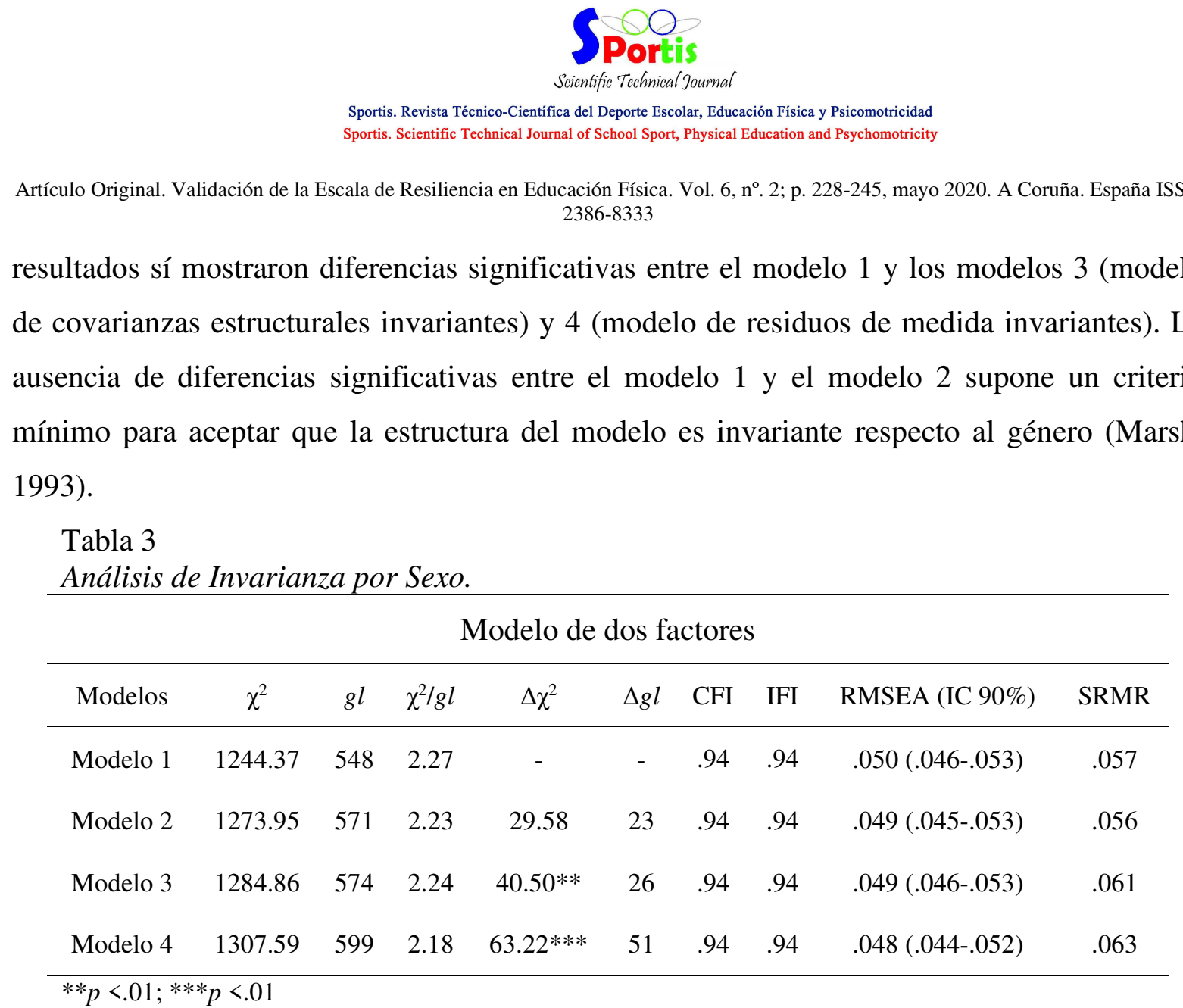

\section{Análisis de fiabilidad y de estabilidad temporal}

Con el fin de obtener evidencias de la fiabilidad de la escala se realizó un análisis de consistencia interna a través de la prueba del $\alpha$ de Cronbach donde las puntuaciones fueron satisfactorias, siendo de .95 para competencia personal y de .96 para aceptación de sí mismo y del contexto. En cuanto al análisis de estabilidad temporal, se calcularon los coeficientes de correlación intra-clase (CCI) y sus intervalos de confianza (IC), arrojando una puntuación de $.88(\mathrm{IC}=.87-.94)$ para competencia personal y de $.93(\mathrm{IC}=.88-.95)$ aceptación de sí mismo y del contexto.

\section{Análisis de validez de criterio}

Con el fin de analizar la validez de criterio de la escala de Resiliencia en el ámbito de las clases de EF se realizó un análisis de regresión lineal (tabla 4) por el cual se introdujo como variable dependiente la motivación intrínseca y como variable independiente los factores competencia personal y aceptación de sí mismo y del contexto. Los resultados mostraron que ambos factores predicen de forma significativa la motivación intrínseca con un peso de regresión positivo y proporcionando una varianza explicada del $31 \%$.

\footnotetext{
Para citar este artículo utilice la siguiente referencia: Trigueros, R.; Navarro, N.; Aguilar-Parra, J.M.; Bermejo, R.; Ferrandiz, C. (2020). Validación de la Escala de Resiliencia en Educación Física. Sportis Sci J, 6 (2), 228-245. DOI: https://doi.org/10.17979/sportis.2020.6.2.5245
} 


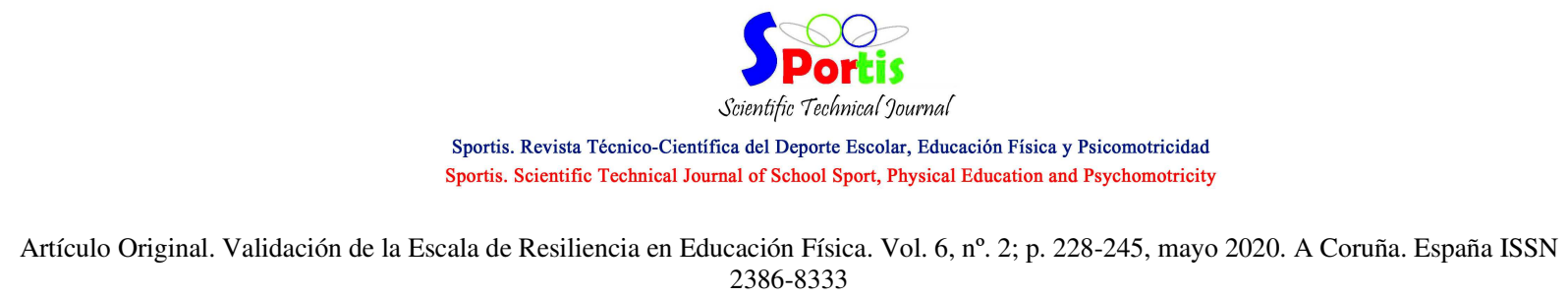

Tabla 4

Análisis de Regresión Lineal.

\begin{tabular}{lcccc}
\hline Variables & $F$ & $R^{2}$ & $\beta$ & $t$ \\
\hline & 116.65 & $.31^{* * *}$ & & \\
Competencia Personal & & & .44 & 10.26 \\
Aceptación de sí mismo y del contexto & & .19 & 4.55 \\
\hline
\end{tabular}

$* * * p<.001$

\section{Discusión}

El objetivo de este estudio fue validar y adaptar la Escala de Resiliencia en el contexto Deportivo de Trigueros, et al, (2017a) al contexto de la EF. La necesidad estriba en el hecho de aumentar la comprensión de las habilidades potenciales de los adolescentes para afrontar experiencias negativas, haciéndoles menos vulnerables a los efectos negativos de los sucesos estresantes. Para ello, resulta necesario el desarrollo y validación de instrumentos que posibiliten el análisis en profundidad de las variables moderadoras que faciliten el desarrollo de respuestas positivas a situaciones de estrés y adversidad.

Los resultados del AFE y AFC han mostrado la existencia de dos factores al igual que la escala original, apoyando la estructura factorial de la escala. El modelo de orden superior presenta unos índices de ajuste adecuados lo que permitirá agrupar los dos factores en uno solo y permitiendo a los investigadores simplificar los modelos estadísticos en futuros estudios, donde el tamaño muestral sea reducido (Trigueros, et al, 2019). El análisis multigrupo mostró que la estructura de la escala se mantenía invariante respecto al sexo, permitiendo por tanto utilizar el instrumento para analizar las posibles diferencias entre chicos y chicas en el ámbito de la EF previamente identificadas por la investigación.

Los resultados del análisis de consistencia interna mostraron unos valores superiores de fiabilidad a .80. Además, el análisis de estabilidad temporal del cuestionario mostró que ambos eran entendidos por la población de forma similar con el paso del tiempo. Por otro lado, la correlación bivariada entre los factores latentes se situaron por debajo de .85. Este Para citar este artículo utilice la siguiente referencia: Trigueros, R.; Navarro, N.; Aguilar-Parra, J.M.; Bermejo, R.; Ferrandiz, C. (2020). Validación de la Escala de Resiliencia en Educación Física. Sportis Sci J, 6 (2), 228-245. DOI: https://doi.org/10.17979/sportis.2020.6.2.5245 


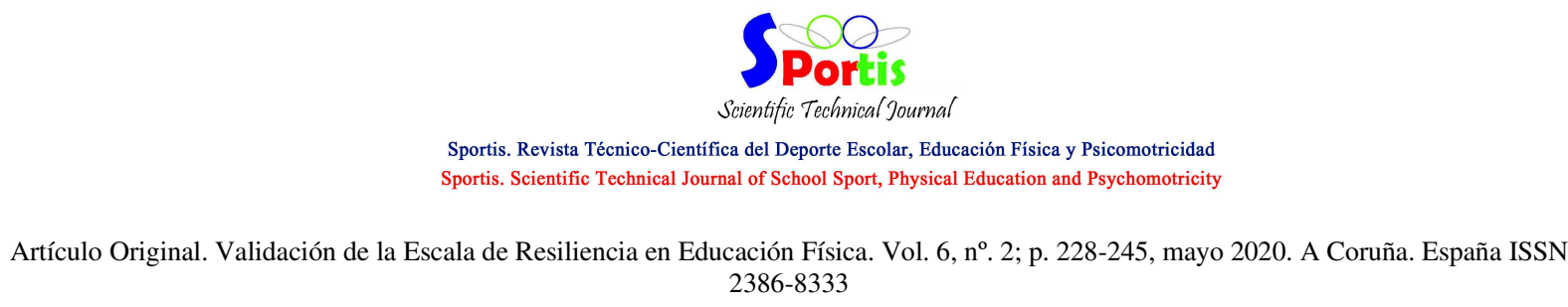

hecho, unido a la existencia de proporciones HTMT en las correlaciones entre los factores que integraban el instrumento fueran inferiores a .85 , sugirió que los factores incluidos en esta nueva versión del instrumento contaban con un adecuado nivel de validez discriminante.

En línea con los resultados de anteriores estudios (Morgan, Fletcher y Sarkar, 2015; Galli y Vealey, 2008), el instrumento ha dado muestras de validez de criterio, ya que, tanto la competencia personal como la aceptación de sí mismo y del contexto se asociaron de forma positiva con la motivación intrínseca. En este sentido, un pleno desarrollo de ambos factores ligados a la resiliencia conllevará la superación de circunstancias adversas y potencialmente estresantes, gracias a elevados y significativos niveles de esfuerzo, de constancia, de dedicación y de sacrificio (Trigueros et al, 2019). Estos aspectos se encuentran ligados a la motivación intrínseca ya que se encuentra asociado con la generación de una serie de conductas adaptativas que favorecen el desempeño del individuo ya que experimentarán mayores niveles de funcionamiento positivo y de ajuste personal (Oliva, et al, 2008; Robertson y Cooper, 2013). En este sentido, un estudio llevado a cabo por Galli y Vealey (2008) en el contexto deportivo ha mostrado como la adversidad, las influencias socioculturales y los recursos personales fueron factores que influían en la resiliencia y que en consecuencia condujeron a resultados positivos hacia su rendimiento y la motivación.

\section{Conclusiones}

De esta forma, los resultados del presente estudio apoyan la utilidad de la nueva versión de la Escala de Resiliencia en el contexto de la Educación Física (EREF) como un instrumento que muestra evidencias de fiabilidad y validez para medir la resiliencia en el contexto español en el ámbito de las clases de EF, además de mostrarse invariante respecto al género. Este instrumento podría contribuir a una mejor comprensión para analizar con más profundidad los procesos mentales y percepciones que tienen los estudiantes durante las clases de EF (Ramírez-Granizo, Alonso-Vargas, Román-Mata, Ubago-Jiménez y MartínezMartínez, 2019) y la influencia que posee en contexto similares (Zurita-Ortega, CastroSánchez, Linares-Manrique y Chacón-Cuberos, 2017). Sin embargo, futuros trabajos deberán analizar con mayor profundidad la robustez del cuestionario con poblaciones y grupos académicos diferentes, con el objetivo de mejorar la herramienta.

Para citar este artículo utilice la siguiente referencia: Trigueros, R.; Navarro, N.; Aguilar-Parra, J.M.; Bermejo, R.; Ferrandiz, C. (2020). Validación de la Escala de Resiliencia en Educación Física. Sportis Sci J, 6 (2), 228-245. DOI: https://doi.org/10.17979/sportis.2020.6.2.5245 


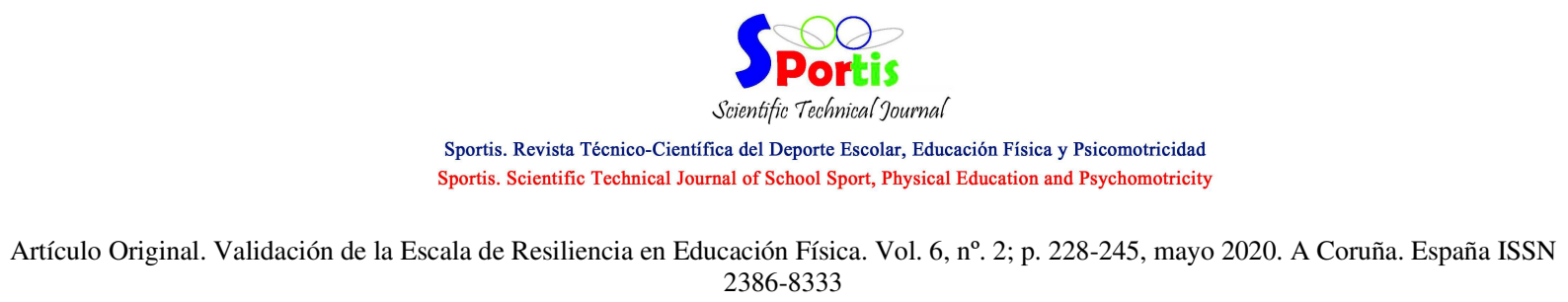

\section{Referencias bibliográficas}

Bamber, M. D. y Schneider, J. K. (2016). Mindfulness-based meditation to decrease stress and anxiety in college students: A narrative synthesis of the research. Educational Research Review, 18, 1-32. Doi: https://doi.org/10.1016/j.edurev.2015.12.004

Baruth, K. E. y Caroll, J. J. (2002). A formal assessment of resilience: The Baruth Protective Factors Inventory. The Journal of Individual Psychology, 58(3), 235-244.

Bernstein, E., Phillips, S. R. y Silverman, S. (2011). Attitudes and perceptions of middle school students toward competitive activities in physical education. Journal of Teaching in Physical Education, 30(1), 69-83. Doi: https://doi.org/10.1123/jtpe.30.1.69

Bryan, C., O'Shea, D. y MacIntyre, T. (2019). Stressing the relevance of resilience: A systematic review of resilience across the domains of sport and work. International Review of Sport and Exercise Psychology, 12(1), 70-111.

Byrne, B. M. (2001). Structural Equation Modeling with AMOS: Basic Concepts, Applications, and Programming. Mahwah, Nueva Jersey: Lawrence Erlbaum Associates.

Cardoso, F. L. y Sacomori, C. (2014). Resilience of athletes with physical disabilities: A cross-sectional study. Revista de Psicología del Deporte, 23(1), 15-22.

Connor, K. M. y Davidson, J. R. (2003). Development of a new resilience scale: The Connor-Davidson resilience scale (CD-RISC). Depression and anxiety, 18(2), 76-82.

Fayers, P. M. y Machin, D. (2000). Quality of life: Assessment, analysis and interpretation. Nueva York: John Wiley.

Fletcher, D. y Sarkar, M. (2012). A grounded theory of psychological resilience in Olympic champions. Psychology of sport and exercise, 13(5), 669-678. Doi: https://doi.org/10.1016/j.psychsport.2012.04.007

Fletcher, D. y Sarkar, M. (2013). Psychological resilience: A review and critique of definitions, concepts, and theory. European Psychologist, 18(1), 12-19. Doi: https://doi.org/10.1027/1016-9040/a000124

Galli, N. y Vealey, R. S. (2008). "Bouncing back” from adversity: Athletes' experiences of resilience. The Sport Psychologist, 22(3), 316-335.

Para citar este artículo utilice la siguiente referencia: Trigueros, R.; Navarro, N.; Aguilar-Parra, J.M.; Bermejo, R.; Ferrandiz, C. (2020). Validación de la Escala de Resiliencia en Educación Física. Sportis Sci J, 6 (2), 228-245. DOI: https://doi.org/10.17979/sportis.2020.6.2.5245 
Hair, J. F. Black, W. C., Babin, B. J. Anderson, R. E. y Tatham, R. L. (2006). Multivariate data analysis. Upper Saddle River, NJ: Pearson

Heath, M. A., Donald, D. R., Theron, L. C. y Lyon, R. C. (2014). AIDS in South Africa: Therapeutic interventions to strengthen resilience among orphans and vulnerable children. School Psychology International, 35(3), 309-337. Doi: https://doi.org/10.1177/0143034314529912

Kohl III, H. W. y Cook, H. D. (Eds.). (2013). Educating the student body: Taking physical activity and physical education to school. National Academies Press.

Kumar, S. y Bhukar, J. P. (2013). Stress level and coping strategies of college students. Journal of Physical Education and Sport Management, 4(1), 5-11.

Lonsdale, C., Rosenkranz, R. R., Peralta, L. R., Bennie, A., Fahey, P. y Lubans, D. R. (2013). A systematic review and meta-analysis of interventions designed to increase moderate-to-vigorous physical activity in school physical education lessons. Preventive medicine, 56(2), 152-161. Doi: https://doi.org/10.1016/j.ypmed.2012.12.004

Lundman, B., Strandberg, G., Eisemann, M., Gustafson, Y. y Brulin, C. (2007). Psychometric Properties of the Version of the Resilience Scale. Scandinavian Journal of Caring Sciences, 21(2), 229-237. Doi: https://doi.org/10.1111/j.1471-6712.2007.00461.x

Madewell, A. N. y Ponce-García, E. (2016). Assessing resilience in emerging adulthood: The resilience scale (RS), Connor-Davidson resilience scale (CD-RISC), and scale of protective factors (SPF). Personality and Individual Differences, 97, 249-255.

Marsh, H. W. (1993). Relations between global and specific domains of self: The importance of individual importance, certainty, and ideals. Journal of Personality and Social Psychology, 65(5), 975-992. Doi: https://doi.org/10.1037/0022-3514.65.5.975

McNair, O. S., Gipson, J. A., Denson, D., Thompson, D. V., Sutton, M. Y. y Hickson, D. A. (2018). The associations of resilience and HIV risk behaviors among black gay, bisexual, other men who have sex with men (MSM) in the Deep South: The MARI study. AIDS and Behavior, 22(5), 1679-1687.

Min, J. A., Yu, J. J., Lee, C. U. y Chae, J. H. (2013). Cognitive emotion regulation strategies contributing to resilience in patients with depression and/or anxiety disorders. 
Artículo Original. Validación de la Escala de Resiliencia en Educación Física. Vol. 6, nº 2; p. 228-245, mayo 2020. A Coruña. España ISSN $2386-8333$

Comprehensive psychiatry, 54(8), 1190-1197. Doi: https://doi.org/10.1016/j.comppsych.2013.05.008

Morgan, P. B., Fletcher, D. y Sarkar, M. (2015). Understanding team resilience in the world's best athletes: A case study of a rugby union World Cup winning team. Psychology of Sport and Exercise, 16, 91-100. Doi: https://doi.org/10.1016/j.psychsport.2014.08.007

Oliva, A., Jiménez, J. M., Parra, A. y Sánchez, M. I. (2008). Acontecimientos vitales estresantes, resiliencia y ajuste adolescente. Revista de Psicopatología y Psicología Clínica, 13(1), 53-62.

Oshio, A., Kaneko, H., Nagamine, S. y Nakaya, M. (2003). Construct validity of the adolescent resilience scale. Psychological reports, 93(3), 1217-1222. Doi: https://doi.org/10.2466/PR0.93.7.1217-1222

Ramírez-Granizo, I., Alonso-Vargas, J. M., Román-Mata, S., Ubago-Jiménez, J. L. y Martínez-Martínez, A. (2019). El rol de la resiliencia en la asociación entre la actividad física deportiva y aspectos académicos en escolares. Sportis, 5(3), 513-531.

Resnick, B. (2018). The relationship between resilience and motivation. In Resilience in Aging (pp. 221-244). Springer, Cham.

Richardson, G. E. (2002). The metatheory of resilience and resiliency. Journal of Clinical Psychology, 58(3), 307-321. Doi: https://doi.org/10.1002/jclp.10020

Rodríguez, M., Pereyra, M. G., Gil, E., Jofré, M., De Bortoli, M. y Labiano, L.M. (2009). Propiedades psicométricas de la escala de resiliencia versión argentina. Evaluar, 9, 7282.

Robertson, I. y Cooper, C. L. (2013). Resilience. Stress and health, 29(3), 175-176. Doi: https://doi.org/10.1002/smi.2512

Schneider, T. R., Lyons, J. B. y Khazon, S. (2013). Emotional intelligence and resilience. Personality and Individual Differences, 55(8), 909-914. Doi: https://doi.org/10.1016/j.paid.2013.07.460

Southwick, S. M., Bonanno, G. A., Masten, A. S., Panter-Brick, C. y Yehuda, R. (2014). Resilience definitions, theory, and challenges: interdisciplinary perspectives. European Journal of Psychotraumatology, 5(1), 25338. Doi: https://doi.org/10.3402/ejpt.v5.25338

Para citar este artículo utilice la siguiente referencia: Trigueros, R.; Navarro, N.; Aguilar-Parra, J.M.; Bermejo, R.; Ferrandiz, C. (2020). Validación de la Escala de Resiliencia en Educación Física. Sportis Sci J, 6 (2), 228-245. DOI: https://doi.org/10.17979/sportis.2020.6.2.5245 
Sundblad, G. B., Jansson, A., Saartok, T., Renström, P. y Engström, L. M. (2008). Self-rated pain and perceived health in relation to stress and physical activity among schoolstudents: A 3-year follow-up. Pain, 136(3), 239-249. Doi: https://doi.org/10.1016/j.pain.2007.06.032

Tavakolizadeh, J., Yazdi, E. S. y Akbary, A. (2019). The Impact of Adler Lifestyle Education on Resiliency of Parents of Educable Mentally Retarded Students. BRAIN. Broad Research in Artificial Intelligence and Neuroscience, 10(3), 5-11.

Thompson, S. R. y Dobbins, S. (2018). The applicability of resilience training to the mitigation of trauma-related mental illness in military personnel. Journal of the American Psychiatric Nurses Association, 24(1), 23-34.

Trigueros, R., Aguilar-Parra, J. M., Cangas-Díaz, A. J., Fernández-Batanero, J. M., Mañas, M. A., Arias, V. B. y López-Liria, R. (2019). The influence of the trainer on the motivation and resilience of sportspeople: A study from the perspective of selfdetermination theory. PloS one, 14(8), e0221461. Doi: https://doi.org/10.1371/journal.pone.0221461

Trigueros, R., Álvarez, J.F., Aguilar-Parra, J.M., Alcaráz-Ibañez, M. y Rosado, A. (2017). Validación y adaptación española de la escala de resiliencia en el contexto deportivo (ERCD). Psychology, Society, \& Education, 9(2), 311-324. Doi: https://doi.org/10.25115/psye.v9i2.864

Trigueros, R., Sicilia, A., Alcaráz-Ibáñez, A. y Dumitru, D.C. (2017). Adaptación y Validación Española de la Escala Revisada del Locus Percibido de Causalidad (PLOC-R) en Educación Física. Cuadernos de Psicología del Deporte, 17(1), 25-32.

Vanhove, A. J., Herian, M. N., Perez, A. L., Harms, P. D. y Lester, P. B. (2016). Can resilience be developed at work? A meta-analytic review of resilience-building programme effectiveness. Journal of Occupational and Organizational Psychology, 89(2), 278-307.

Vlachopoulos, S. P., Katartzi, E. S., Kontou, M. G., Moustaka, F. C. y Goudas, M. (2011). The revised Perceived Locus of Causality in Physical Education Scale: Psychometric evaluation among youth. Psychology of Sport and Exercise, 12, 583-592. Doi: https://doi.org/10.1016/j.psychsport.2011.07.003

Para citar este artículo utilice la siguiente referencia: Trigueros, R.; Navarro, N.; Aguilar-Parra, J.M.; Bermejo, R.; Ferrandiz, C. (2020). Validación de la Escala de Resiliencia en Educación Física. Sportis Sci J, 6 (2), 228-245. DOI: https://doi.org/10.17979/sportis.2020.6.2.5245 
Artículo Original. Validación de la Escala de Resiliencia en Educación Física. Vol. 6, nº 2; p. 228-245, mayo 2020. A Coruña. España ISSN 2386-8333

Wagnild, G. y Young, H. M. (1993). Development and psychometric evaluation of the Resilience Scale. Journal of Nursing Measurement, 1(2), 165-178.

Wagnild, G.M. (2009). A review of resilience scale. Journal of Nursing Measurement, 17, 105-13. Doi: https://doi.org/10.1891/1061-3749.17.2.105

Zurita-Ortega, F., Castro-Sánchez, M., Linares-Manrique, M. y Chacón-Cuberos, R. (2017). Resiliencia, un elemento de prevención en actividad física. Sportis, 3(1), 50-62. 